\title{
GENERIC WELL-POSEDNESS IN MINIMIZATION PROBLEMS
}

\author{
A. IOFFE AND R. E. LUCCHETTI
}

Received 12 February 2004

The goal of this paper is to provide an overview of results concerning, roughly speaking, the following issue: given a (topologized) class of minimum problems, "how many" of them are well-posed? We will consider several ways to define the concept of "how many," and also several types of well-posedness concepts. We will concentrate our attention on results related to uniform convergence on bounded sets, or similar convergence notions, as far as the topology on the class of functions under investigation is concerned.

\section{Introduction}

Given a space $X$, and an (extended) real-valued function $f: X \rightarrow(-\infty, \infty]$, we consider the abstract minimization problem: find

$$
x \in X: f(x)=\inf f .
$$

Set $\operatorname{Min}(f)=\{x \in X: f(x)=\inf f\}$ and $f^{a}=\{x: f(x) \leq a\}$. Observe that

$$
\operatorname{Min} f=\bigcap_{a>\inf f} f^{a}
$$

The most important existence theorem, probably one of the most elegant theorems in analysis, is the Weierstrass theorem, which can be stated in the following way.

Theorem 1.1. Suppose there are a topology $\tau$ on $X$ and $\bar{a}>\inf f$ such that

(i) $f^{a}$ is $\tau$-closed for all $a \leq \bar{a}$;

(ii) $f^{\bar{a}}$ is $\tau$-compact.

Then the minimum problem has a solution, that is, $\operatorname{Min} f$ is nonempty.

Its proof is marvellously simple. Min $f$ is the intersection of a nested family of (nonempty) closed sets, and one of them is compact. Then it is nonempty.

A useful variant of the proof can be given by considering minimizing sequences, that is, sequences $\left\{x_{n}\right\} \subset X$ such that $f\left(x_{n}\right) \rightarrow \inf f$ (of course there are always such sequences). As $x_{n} \subset f^{a}$ eventually, then it must have a converging subsequence. Now closedness of $f^{a}$, for all $a \leq \bar{a}$, implies that every limit point of $\left\{x_{n}\right\}$ minimizes $f$. The challenge is, 
given a specific problem, to find a suitable topology $\tau$, in order to apply the theorem. As it is obvious, and also very natural, the two assumptions - having closed level sets, having one of them compact-go in opposite directions: a topology rich in open (and so closed) sets usually is poor in compact sets. So, what can be said in general when a topology with sufficiently many compact sets is not available? Surely, no existence theorem as general as that provided by Weierstrass a little more than one century ago can be proved. Thus, it becomes interesting to consider classes of problems, and to prove that inside a certain class those having solutions are sufficiently many. For instance, they contain a dense set. From the point of view of applications, this would not be so bad, since the performance function to be minimized is usually known up to some approximation errors, and thus it would not be too costly to change it a bit (in a suitable way).

However, it is interesting to go further and to ask for more stringent properties than just density. Other notions of "sufficiently many" are of interest. In this paper, we will mainly consider properties related to category, in the sense of Baire, and to the alternative notion of $\sigma$-porosity, which will be discussed in the following. In a finite-dimensional setting, we will also consider the idea of full measure sets.

Actually, not only do we expect a problem to have a solution, maybe unique, but also this solution is "easy to find." This leads to the idea(s) of well-posedness, that will be considered in the sequel. Thus, our program can be outlined in the following (somewhat loose) way: to consider several different classes of minimization problems, and inside them to prove that "very many" problems "have solutions and are easy to solve." To start with quoting results in this sense, interesting results about generic convergence of descent methods can be found in $[17,18]$.

A first efficient way to tackle this topic is to rely on some variational principles. Probably the first one, and the most famous, is the Ekeland variational principle, which itself offers a result of dense existence, and furthermore provides a basis on which to build up several other results. Thus we will start with an overview of some variational principles, especially those by Ekeland, Deville-Godefroy-Zizler, as well as two more recent ones, from Ioffe-Zaslavski and from Revalski and the authors. The core of the paper will then be dedicated to a review of well-posedness results for various classes of optimization problems.

\section{Variational principles}

We start with the first, and most famous, variational principle: the Ekeland principle.

Theorem 2.1. Let $(X, \rho)$ be a complete metric space and let $f: X \rightarrow(-\infty, \infty]$ be a lower semicontinuous, lower bounded function. Let $\varepsilon>0, r>0$, and $\bar{x} \in X$ be such that $f(\bar{x}) \leq$ $\inf _{X} f+r \varepsilon$. Then, there exists $\hat{x} \in X$ enjoying the following properties:

(1) $\rho(\hat{x}, \bar{x}) \leq r$;

(2) $f(\hat{x}) \leq f(\bar{x})-\varepsilon \rho(\bar{x}, \hat{x})$;

(3) $f(\hat{x})<f(x)+\varepsilon \rho(\hat{x}, x)$ for all $x \neq \hat{x}$.

This beautiful result has an enormous number of interesting and sometimes surprising consequences (e.g., it is possible to derive from it the famous mountain pass theorem 
by Ambrosetti and Rabinowitz), but we immediately focus on one of them which is of interest to us in the paper.

Condition (3) above implies a density result for problems with a unique solution. We explain why. Consider, for simplicity, the space $\mathscr{F}$ of the real valued, lower semicontinuous positive functions on the complete metric space $(X, \rho)$. We endow $\mathscr{F}_{F}$ with a distance compatible with uniform convergence on bounded sets. For instance, we pick any element $\theta \in X$, and set, for any two $f, g \in \mathscr{F}$ and $n \in \mathbb{N}$,

$$
\|f-g\|_{n}=\sup _{\rho(x, \theta) \leq n}|f(x)-g(x)|
$$

If $\|f-g\|_{n}=\infty$ for some $n$, then we set $d(f, g)=1$. Otherwise,

$$
d(f, g)=\sum_{n=1}^{\infty} 2^{-n} \frac{\|f-g\|_{n}}{1+\|f-g\|_{n}} .
$$

It is easy to see that in such a way $(\mathscr{F}, d)$ is a complete metric space.

We can now state the following proposition.

Proposition 2.2. In $(\mathscr{F}, d)$ the set of functions attaining the minimum value at a unique point is dense.

Proof. Fix $\sigma>0$, take $j$ so large that, setting $g(x)=f(x)+(1 / j) \rho(x, \theta)$, we have $d(f, g)<$ $(\sigma / 2)$. Now, observe that $\lim _{\rho(x, \theta) \rightarrow \infty} g(x)=\infty$, and thus there exists $M$ such that $g^{1} \subset$ $B(\theta, M)$. Let $s=\sum\left(1 / 2^{n}\right)(n+M)$. Apply the principle with $\varepsilon=(\sigma / 2 s)(r$ arbitrary) to find $\hat{x}$ such that $\rho(\hat{x}, \theta) \leq M$ and $\hat{x}$ is the unique minimizer of

$$
h(\cdot)=g(\cdot)+\varepsilon \rho(\cdot, \hat{x}) .
$$

As $|h(x)-g(x)|_{n} \leq \varepsilon(n+M)$, it follows that $d(h, g) \leq \varepsilon s=(\sigma / 2)$. Then $d(f, h)<\sigma$, and the proof is complete.

This is just an example of how to use the Ekeland principle to get such a type of results. It is possible to get similar results for other classes of functions and other topologies: we will see some examples later.

The requirement of having existence and uniqueness of the minimizer can be strengthened. The following definition is widely used in the literature.

Definition 2.3. Let $(X, \rho)$ be a metric space, let $f: X \rightarrow(-\infty, \infty]$ be lower semicontinuous. Then $(X, f)$ (or simply $f$ ) is said to be Tykhonov well-posed if

(1) there exists a unique $\bar{x} \in X$ such that $f(\bar{x}) \leq f(x)$ for all $x \in X$;

(2) every sequence $\left\{x_{n}\right\}$ such that $f\left(x_{n}\right) \rightarrow \inf _{X} f$ is such that $x_{n} \rightarrow \bar{x}$.

For some purposes, sometimes the uniqueness of the solution is a too restrictive assumption. Thus a definition of Tykhonov well-posedness in extended sense can be given by requiring compactness of the solution set, rather than uniqueness of the minimizer, and, consequently, convergence of minimizing sequences up to subsequences. Thus, if 
there exists $a>\inf f$ such that $f^{a}$ is compact, then $f$ is well-posed in the generalized sense. This means that in the assumption of Weierstrass theorem, the problem is actually well-posed in extended sense. We will provide later other definitions of well-posedness, by requiring uniqueness of the minimizer: it is intended that the same adjustments can be done in all cases, exactly as in the above one.

Just to give some examples of well-posed problems, a convex, lower semicontinuous extended real-valued function on a Euclidean space, with unique minimizer, gives rise to a Tykhonov well-posed problem (this is no longer true in infinite dimensions). The Tykhonov well-posedness of the best approximation problem, minimize $\|x-\hat{x}\|$ on a given closed convex set $C \subset X$, depends on the structure of the underlying Banach space $X$ : it is well-posed, for every $\hat{x} \in X$ and $C \subset X$ if and only if $X$ is a so-called E-space, that is, it is reflexive, strictly convex (the boundary of the unit ball contains no line segments) and fulfills the so-called Kadeč-Klee property $\left(x_{n}-x\right.$ and $\left\|x_{n}\right\| \rightarrow\|x\|$ imply $\left.x_{n} \rightarrow x\right)$. The best approximation problem is a very important one in optimization, thus also its generic well-posedness has been studied, see $[6,19]$.

The next proposition, due to Furi and Vignoli, provides a useful characterization of Tykhonov well-posedness, and is largely used in proving genericity results.

Proposition 2.4. Let $X$ be a complete metric space and let $f: X \rightarrow(-\infty, \infty]$ be a lower semicontinuous function. The following are equivalent:

(i) $f$ is well-posed;

(ii) $\inf _{a>\inf f} \operatorname{diam} f^{a}=0$.

A classical pattern to prove that, in a given class $(\mathscr{F}, d)$ of functions such that $(\mathscr{F}, d)$ is a complete metric space, or at least a Baire space, the well-posed problems are a large set, that is, contain a dense $G_{\delta}$ set, is to define

$$
V_{n}:=\left\{f \in \mathscr{F}: \inf _{a>\inf f} \operatorname{diam} f^{a}<\frac{1}{n}\right\}
$$

and to prove that each $V_{n}$ is an open set (i.e., upper semicontinuity of the map $f \mapsto$ $\operatorname{diam} f^{a}$ ), then an ad hoc use of the Ekeland principle and the Furi-Vignoli criterion allows concluding.

But we come back to the variational principles. The following one, due to DevilleGodefroy-Zizler, has, among its consequences, the possibility to prove genericity results for Tykhonov well-posed problems. Remember that a bump function on a space $X$ is a function with bounded support.

Theorem 2.5. Let $X$ be a Banach space admitting a Lipschitz and Fréchet differentiable bump function. Then for every lower semicontinuous, bounded from below, function $f: X \rightarrow$ $(-\infty, \infty]$, and for every $\varepsilon>0$, there exists a Lipschitz and Fréchet differentiable function $g$ such that $\|g\|_{\infty}<\varepsilon,\left\|g^{\prime}\right\|_{\infty}<\varepsilon$, and $f+g$ is Tykhonov well-posed.

It is not difficult to see that the Ekeland variational principle, as far as the third condition is concerned, can be derived from the previous one, however, it is not possible in this way to locate the minimum point with the same accuracy (condition (1)), see [12]. It must be also noticed that it implies the stronger condition of Tykhonov well-posedness. 
Anyway, though rather general, the above principle does not apply to several interesting situations, for instance, when convex functions are involved, it is clear that perturbations made by bump functions can kill convexity. Thus, Ioffe and Zaslavski provided in [13] a new principle, aimed at getting sharper results and suited to more general classes of functions. Moreover, they involve a more stringent notion of well-posedness, and so the results derived from their principle are sharper. The required setting is as follows.

Let $(X,\|\cdot\|)$ be a real Banach space and let $(\mathscr{A}, d)$ be a metric space which is a Baire space. We will call $X$ the domain space. The space $\mathscr{A}$ will serve as a data space. Assume that with each $a \in \mathscr{A}$, a lower semicontinuous extended real-valued function $f_{a}: X \rightarrow$ $\mathbb{R} \cup\{+\infty\}$ is associated and consider the problem of minimizing $f_{a}$ on $X$. Denote by inf $f_{a}$ the infimum of $f_{a}$ on the space $X$.

We say that this problem (for a given $a$ ) is well-posed, provided

(1) inf $f_{a}$ is finite and attained at a unique point $x_{0} \in X$;

(2) for any sequence $\left\{a_{n}\right\}$ converging to $a$, inf $f_{a_{n}}$ is finite for large $n$ and any sequence $\left\{z_{n}\right\} \subset X$ such that $f_{a_{n}}\left(z_{n}\right)-\inf f_{a_{n}} \rightarrow 0$ strongly converges to $x_{0} ;$

(3) if $a_{n}$ converges to $a$, then inf $f_{a_{n}} \rightarrow \inf f_{a}=f_{a}\left(x_{0}\right)$.

The first two conditions are called "well-posedness by perturbations" in [23, 24], while the third one is known in the literature as value Hadamard well-posedness (see, e.g., [8]).

Now, consider the following condition:

( $\mathscr{H}$ ) there is a dense subset $\mathscr{B} \subset \mathscr{A}$ such that for any $a \in \mathscr{B}$, any $\varepsilon>0$, and $\gamma>0$, there exist a nonempty open set $\mathscr{V} \subset \mathscr{A}, \bar{x} \in X, \alpha \in \mathbb{R}$, and $\lambda>0$ such that for every $b \in \mathscr{V}$ we have

(i) $d(a, b)<\varepsilon$ and inf $f_{b}>-\infty$;

(ii) if $z \in X$ is such that $f_{b}(z)<\inf f_{b}+\lambda$, then $\|z-\bar{x}\| \leq \gamma$ and $\left|f_{b}(z)-\alpha\right| \leq \gamma$.

The variational principle from [13] can now be stated as follows.

Theorem 2.6 [13]. Let $X$ be a real Banach space and let $(\mathscr{A}, d)$ be a Baire space. Suppose (H) holds. Then there exists a dense $G_{\delta}$-subset $\mathscr{A}_{1}$ of $(\mathscr{A}, d)$ such that for every a $\in \mathscr{A}_{1}$, the corresponding minimization problem is well-posed.

In the following section, we will mention several results that can be derived from the above principle. We only notice here that the Deville-Godefroy-Zizler principle is a straightforward consequence of the previous one.

The Ioffe-Zaslavski principle is suited to prove generic well-posedness (in the sense of Baire category), for a broad variety of topologies. But very often, and typically in the well-posedness context, a genericity result is not fully satisfactory.

The reason is, intuitively, that the idea of "smallness" has quantitative connotation and is naturally associated with concepts similar to "measure zero" or "probability zero" (see, e.g., [20]), while a meager set in the sense of Baire already in $\mathbb{R}$ can have a positive (even infinite) measure.

The idea of porosity offers a perfect way out of the measure-category dichotomy, since $\sigma$-porous sets are always sets of the first Baire category and, in Euclidean spaces, of Lebesgue measure zero.

Definition 2.7. Let $(X, d)$ be a metric space and $A \subset X$. The set $A$ is called porous in $X$ if there are $\lambda \in(0,1)$ and $r_{0}>0$ such that for any $x \in A$ and $r \in\left(0, r_{0}\right)$, there is $y \in X$ such 
that $B(y, \lambda r) \subset B(x, r) \backslash A$. $A$ is called $\sigma$-porous in $X$ if it is a countable union of porous sets in $X$.

In fact, the above definition is stronger than the original (local) definition given in [21], where a set as above is called "uniformly very porous."

We can now state the new principle as follows (see [11]).

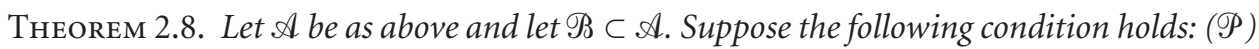
for any $k \in \mathbb{N}$, there are $\lambda=\lambda(k) \in(0,1)$ and $\varepsilon_{0}=\varepsilon_{0}(k)>0$ such that for each $a \in \mathscr{B}$ and each $\varepsilon \in\left(0, \varepsilon_{0}\right)$, there exist $\bar{a}=\bar{a}(k, a, \varepsilon) \in \mathscr{A}$ and $\eta>0$ with the following properties:

(i) $B(\bar{a}, \lambda \varepsilon) \subset B(a, \varepsilon)$;

(ii) $b \in B(\bar{a}, \lambda \varepsilon) \Rightarrow \inf f_{b}>-\infty \& \operatorname{diam}\left(\bigcup_{b \in B(\bar{a}, \lambda \varepsilon)} f_{b}^{\eta}\right)<1 / k$.

Then the set $\{a \in \mathscr{B}: a$ is not well-posed $\}$ is $\sigma$-porous in $\mathcal{A}$.

Actually, we find it convenient to use, in our applications, the following corollary.

Corollary 2.9. Suppose $\mathscr{A}=\bigcup_{m=0}^{\infty} \mathscr{A}_{m}$, where $\mathscr{A}_{0}$ is a $\sigma$-porous set in $\mathscr{A}$, and that, for each $m \geq 1$, condition $(\mathscr{P})$ holds for $\mathscr{B}=\mathscr{A}_{m}$. Then the set of well-posed problems inside $\mathscr{A}$ has a $\sigma$-porous complement in $\mathscr{A}$.

To end this section, we will mention, without going in further details, other variational principles: first of all, that one provided by Borwein-Preiss, suited to deal also with smooth perturbations, then that one provided by Revalski and the authors [10]. The latter is suited to give a unified approach to get results for problems with functional constraints, which will be described in the next section. Finally, an extension of the DevilleGodefroy-Zizler principle, involving $\sigma$-porosity rather than Baire category, has been proved by Deville-Revalski in [7].

\section{Genericity results}

In this section, we collect several genericity results for well-posed problems. We remind that we will be concerned with the topology of the uniform convergence on bounded sets, or similar ones. In particular, we will not pay attention to results dealing with uniform convergence on the entire space, or finer. When the results are consequence of the principles described in the previous section, we will mention this explicitly. Otherwise, it is intended that the results are obtained with direct proofs.

We will start with a short and quick survey of older results. Later, we will describe in more details more recent results.

Probably the first genericity result can be found in [14]. Denote by $\Gamma(X)$ the set of the extended real-valued convex, lower semicontinuous functions on the Banach space $X$, and by $\widetilde{\Gamma}(X)$ its subset consisting of the real-valued functions. The first result reads as.

Theorem 3.1. Let $X$ be a reflexive Banach space, and endow $\tilde{\Gamma}(X)$ with the uniform convergence on bounded sets. Then the Tykhonov well-posed problems are a dense $G_{\delta}$ subset of $\widetilde{\Gamma}(X)$.

The proof relies on the above-mentioned characterization by Furi and Vignoli of Tykhonov well-posed problems (see Proposition 2.4). 
It should be noticed that for many purposes in $\Gamma(X)$, it is more natural to consider the well-known Mosco convergence. A negative result however holds in this case: if $X$ is infinite dimensional, then the family of the functions which are unbounded from below is a dense $G_{\delta}$ set (see [4]). A positive result in this class was proved instead, in the same paper, with another important set convergence notion. Endow $\Gamma(X)$ with the bounded Hausdorff topology (thus making it a completely metrizable space). Then the family of the well-posed functions is a dense $G_{\delta}$ subset of $\Gamma(X)$ (see Theorem 4.8).

For the reader not acquainted with the bounded Hausdorff metric topology, we just mention that on a space of closed convex sets, bounded Hausdorff convergence of a sequence of sets is equivalent to Hausdorff convergence when intersecting all sets with all (large) balls, and when applied to functions, it is intended that we look at the convergence of the epigraphs. In the general case, the definition looks a bit more complicated (see, e.g., [2]).

A similar result has been obtained by Ioffe-Zaslavski, which also proves the same genericity statement for the class of the quasiconvex lower semicontinuous functions (thus extending to infinite dimensions a previous result by Beer and Lucchetti, see [3]) and for the class of the lower semicontinuous functions minorized by a fixed coercive function (see [13, Section 4]). The principle is used in the same paper in order to obtain an interesting result in the calculus of variations. Namely, it is shown that, for a suitable topology, in the space of the normal integrands, without either convexity or growth conditions, the majority of the problems are well-posed. It should be noticed that the theorem fails as far as only autonomous integrands are considered, as it can be shown that, around the classical Bolza functional, for which there is no minimizer, all (autonomous) problems either do not have solutions, or else have more than one solution (see [9, Theorem 5]).

We now switch to constrained problems. A typical one is to minimize a given function $f$ over a constraint set $A$, problem denoted by $(A, f)$. The first result in this direction is as follows: denote by $(C(X), \tau)$ the family of the closed convex subsets of a Banach space $X$, endowed with the bounded Hausdorff topology, and consider the space $\Gamma(X)$ defined above, endowed always with the bounded Hausdorff topology. Then $C(X) \times \Gamma(X)$ becomes a complete metric space. Inside this space, the Tykhonov well-posed problems are a dense $G_{\delta}$ set (see [5, Theorem 5.4]). Again, the density part is derived from the Ekeland variational principle, while the Furi-Vignoli characterization provides the rest of the proof. It should be noticed that a notion stronger than Tykhonov well-posedness is actually proved, though this result is still weaker than Ioffe and Zavslaski's one. They prove the same theorem (in [13]) as well as one applying the nonconvex case. Their main condition is that the functions in the considered family have to be minorized by the same coercive function.

We now turn our attention to other more recent results.

First of all, we mention some of those derived in [9] from the Ioffe-Zaslavski principle. Let $\mathscr{A}_{c}$ denote the set of the convex functions defined on a Banach space $X$, realvalued and continuous, and let $A_{q c}$ denote the set of the quasiconvex functions defined on a Banach space $X$, real-valued, continuous, and lower bounded on the bounded sets. We topologize both sets with the topology of the uniform convergence on bounded sets, 
and consider the following programming problem:

(P)

$$
\text { minimize } f_{0}(x) \text { subject to } f_{i}(x) \leq 0, i=1, \ldots, k \text {. }
$$

A typical element of the data space then will be $a=\left(f_{0}, \ldots, f_{k}\right)$, where $a \in A_{c}^{k+1}$ (resp., $\left.a \in A_{q c}^{k+1}\right)$. We denote by $\mathscr{F}(a)$ the feasible set of the problem. Clearly, we cannot expect $\mathscr{F}(a)$ to be generically nonempty, so we will assume that our data space $\mathscr{A}$ will be the closure of $\{a: \mathscr{F}(a) \neq \varnothing\}$ (a Baire space). We then have the following theorem.

Theorem 3.2. The problem $(P)$ is generically well-posed in $A$ for every Banach space $X$, as far as the convex case is concerned, and the same is true for the quasiconvex case, provided the space $X$ is reflexive.

Coming back to the Ioffe-Zaslavski principle, we see that, in order to apply property ( $\mathscr{H}$ ), we first require a suitable dense subset $\mathscr{B}$ such that, for any element $a \in \mathscr{B}$ and $\varepsilon>0$, it is possible to find an open set $U$ such that $U$ is contained in the $\varepsilon$ ball around $a$ and all elements of $U$ have all their level sets of some prescribed height close to a given point. Then one has to specify both the set $\mathscr{B}$ and, for each element $a$ in $B$, the open set $U$, usually a small ball around $a$. In the convex case, $\mathscr{B}$ is given as the set of those problems for which the objective function $f_{0}$ is coercive and, for each element in $B$, the center of the close-by ball corresponds to the perturbation of the objective function and the constraints, made by adding a term of the form $\tau\|x-\bar{x}\|$ to the objective and of the form $\tau\|x-\bar{x}\|-(\tau / 2)$ to the constraints, with $\bar{x}$ and $\tau$ suitably chosen. It is clear that, as far as the objective function is concerned, the term $\tau\|x-\bar{x}\|$ creates a "narrow well" that allows getting the required conditions on the level sets of nearby functions. The perturbations on the constraint functions are suited to ensure that the point $\bar{x}$ is feasible for nearby problems, and this allows making some estimates on the values of the close-by problems. More or less the same idea is used in the quasiconvex case, but then the construction is much more intricate, as perturbations like those used in the convex case are not allowed with quasiconvexity. So we do not describe them here, and instead refer the interested reader to the original paper [9].

Generalizations to other problems with functional constraints are contained in [10]. We describe them in the next examples, where the conclusion is always the same: in the specified class, the majority of problems are well-posed.

Let $X, Y, Z$ be Banach spaces, let $C$ be a closed convex pointed cone with some element $e \in \operatorname{int} C$. Let $f: X \rightarrow \mathbb{R}$ be a given continuous function, let $F: X \rightarrow Y$ and $G: X \rightarrow Z$ be continuous maps, and consider the following (abstract) minimization problem:

$\left((\mathbf{P})_{a}\right)$

$$
\text { minimize } f(x) \text { subject to } F(x) \in C ; G(x)=0 \text {, }
$$

where $a$ is a data element generating $f, F, G$. To make this clear, we see how this theoretical setting applies to some examples. 
Example 3.3. Consider the following quadratic programming problem with linear equality constraints:

$$
\operatorname{minimize}(Q x \mid x)+(c \mid x) \quad \text { subject to } A x=b \text {, }
$$

where $(\cdot \mid \cdot)$ is the scalar product in $\mathbb{R}^{n}, Q$ is an $n \times n$ symmetric matrix, $A$ is an $m \times n$ matrix, $c \in \mathbb{R}^{n}$, and $b \in \mathbb{R}^{m}$.

It is then natural to set $a=(Q, A, c, b)$ and take $f(x)=(Q x \mid x)+(c \mid x)$ and $G(x)=$ $A x-b$.

Thus every problem $\left((\mathbf{P})_{a}\right)$ can be equivalently represented as an unconstrained minimization of the extended real-valued function

$$
f_{a}(x)= \begin{cases}f(x), & \text { if } F(x) \in C, G(x)=0 ; \\ \infty, & \text { otherwise. }\end{cases}
$$

The feasible set $(\mathrm{MP})_{a}$ coincides with the domain of $f_{a}$ :

$$
\operatorname{dom} f_{a}=\{x \in X: F(x) \in C, G(x)=0\} .
$$

The subset of the data space we will consider in all cases is the set of the meaningful problems, that is,

$$
M=\left\{a:\left|\inf f_{a}\right|<\infty\right\} .
$$

This means that we consider feasible and lower bounded problems.

Once we have proved that $\mathcal{M}$, endowed with an appropriate metric, is a Baire space, we are then in the framework of the Ioffe-Zaslavski principle, from which we derive ours, suited to problems of this kind. We will not detail here our principle, which is a bit technical. We instead set forth a few examples in which this principle does apply, thus providing generic well-posedness inside $M$.

Our first example concerns the standard mathematical programming problem.

Example 3.4. Consider the following problem:

$$
\text { minimize } f(x) \text { subject to } f_{1}(x) \leq 0, \ldots, f_{m}(x) \leq 0, g_{1}(x)=0, \ldots, g_{l}(x)=0 \text {, }
$$

where $f \in C^{k}(X), F(\cdot)=\left(f_{1}(\cdot), \ldots, f_{m}(\cdot)\right) \in C^{k}(X, Y)$ and $G(\cdot)=\left(g_{1}(\cdot), \ldots, g_{l}(\cdot)\right) \in$ $C^{k}(X, Z)$.

Suppose we are given a function $\psi: X \rightarrow \mathbb{R}$, continuous and coercive, that is, $\psi(x) \rightarrow \infty$ as $\|x\| \rightarrow \infty$, and consider the set

$$
C_{\psi}^{k}(X)=\left\{f \in C^{k}(X): f \geq \psi\right\}
$$

with the distance

$$
d_{k b}(f, g)=\sum_{j=0}^{\infty} \frac{1}{2^{j}} \rho_{j k}(f, g),
$$


where the pseudometric $\rho_{j k}(f, g)$ is defined as

$$
\rho_{j k}(f, g)=\sum_{i=0}^{k} \sup \left\{\frac{\left|f^{(i)}(x)-g^{(i)}(x)\right|}{1+\left|f^{(i)}(x)-g^{(i)}(x)\right|}:\|x\| \leq j\right\} .
$$

This distance defines the topology of the uniform convergence of functions and their derivatives up to the order $k$ on the bounded subsets of $X$.

Thus the data space now is

$$
C_{\psi}^{k}(X) \times C^{k}(X) \times \cdots \times C^{k}(X),
$$

with some product metric obtained by the metrics $d_{k b}$ in the component spaces.

In the previous example, the domain space $X$ can be infinite dimensional, provided there exists a positive $C^{k}$-bump function $q(\cdot)$ with minimum at zero and greater than the one outside the unit ball. This is certainly the case in any Banach space if $k=0$, while if $k=1$, a sufficient condition for the existence of such $q$ is that $X$ has an equivalent Fréchet differentiable norm.

Our second example is a generalization of the quadratic programming in Hilbert space, according to Example 3.3.

Example 3.5. Let $X$ be a real Hilbert space with inner product $(\cdot \mid \cdot)$. The class of problems to be considered is described by the following scheme:

$$
\text { minimize }(Q x \mid x)+(c \mid x) \quad \text { subject to }\left(Q_{i} x \mid x\right)+\left(c_{i} \mid x\right) \leq \alpha_{i}, i=1, \ldots, k ; A x=u \text {. }
$$

Here $Q, Q_{i}$ are symmetric bounded linear operators in $X, A$ is a bounded linear operator in $X, c, c_{1}, \ldots, c_{k}$, and $u$ are elements of $X$, and $\alpha_{i}$ are real numbers. Thus a typical $a$ on the data space is a $(3 k+4)$-uple of the form

$$
a=\left(Q, Q_{1}, \ldots, Q_{k}, A, c, c_{1}, \ldots, c_{k}, u, \alpha_{1}, \ldots, \alpha_{k}\right)
$$

which we will consider with the natural product topology corresponding to the norm convergence of operators and vectors of $X$ and the usual convergence of numbers. We assume that $Q, Q_{i}$ are positive semidefinite matrices (an unintentionally missed assumption in the paper) and the operator $A$ maps the Hilbert space $X$ onto itself: $A(X)=X$. Such operators form an open set in the space of bounded operators with the usual operator norm.

The next example concerns semiinfinite programming.

Example 3.6. Let $X=\mathbb{R}^{n}$, let $T$ be a Hausdorff compact space, and consider the following problem:

$$
\text { minimize }(A x \mid x)+(c \mid x) \quad \text { subject to }(B(t) \mid x)+b(t) \leq 0 \text {, }
$$

where $A$ is a real, symmetric $n \times n$ matrix, while $B: T \rightarrow \mathbb{R}^{n}, b: T \rightarrow \mathbb{R}$ are continuous functions on $X$. With the previous notations, the Banach space $Y$ is the space $C(T)$ of 
the real-valued continuous functions on $T$ (with the usual max norm), $C$ is the cone of the functions which are nonpositive everywhere, and $e$ is the constant function-valued -1 . The data space is the set of 4-uples $a=(A, B, c, b)$, equipped with some product metric generated by the usual metrics on matrices $A$ and vectors $c$ and by the sup-norms for $B$ in $C\left(T, \mathbb{R}^{n}\right)$ and for $b$ in $C(T)$.

The last example concerns optimal control problems with quadratic costs.

Example 3.7. This is the class of problems covered by the following scheme:

$$
\begin{aligned}
& \text { minimize } \int_{0}^{1}\left[(P u(t) \mid u(t))+(Q x(t) \mid x(t))+\left(c_{0}(t) \mid u(t)\right)+(b(t) \mid x(t))\right] d t \\
& \text { subject to } \dot{x}(t)=A x(t)+B u(t) ; x(0)=x_{0}, x(1)=x_{1} .
\end{aligned}
$$

Here $P$ and $Q$ are symmetric matrices of orders $m$ and $n$, respectively, with $P$ positively semidefinite, $A$ is a square matrix of order $n, B$ is a matrix $n \times m, x_{0}, x_{1} \in \mathbb{R}^{n}$, and $c_{0}(t)$ and $b(t)$ are square integrable mappings from $[0,1]$ into $\mathbb{R}^{m}$ and $\mathbb{R}^{n}$, respectively. Thus the data space of the problem consists of 8-uples $a=\left(P, Q, c_{0}(\cdot), b(\cdot), A, B, x_{0}, x_{1}\right)$, and may be identified with the product $S L^{+}(m) \times S L(n) \times L_{2}^{m}(0,1) \times L_{2}^{n}(0,1) \times L(n) \times L(m, n) \times \mathbb{R}^{n} \times$ $\mathbb{R}^{n}$ endowed with a natural product metric.

We refer to [22] for other genericity results concerning optimal control problems.

To conclude this section, we remark that some of the previous examples can be given a generic result, using the same principle, but different topologies in the data space (usually, the topology of uniform convergence in the whole space, whenever it makes sense).

\section{Porosity results}

We now switch to porosity results, as obtained in [11]. Observe that in the case of porosity the distance on the data space must be carefully specified, as porosity requires quantitative estimates. As in the previous section, we will always deal with distances inducing uniform convergence on bounded sets.

So, let $(X, \rho)$ be a metric space and $\mathscr{F}$ a linear space of real-valued functions on $X$, endowed with the metric described after Theorem 2.1.

Here is the first result.

Let $(X,\|\cdot\|)$ be a real Banach space, and $\mathscr{F}$ one of the following two spaces of functions on $X$ :

(a) $\mathrm{QC}(X)=$ : the space of all real-valued quasiconvex continuous functions in $X$ bounded from below on bounded sets;

(b) $\operatorname{Conv}(X)=$ : the space of all convex continuous functions on $X$.

Theorem 4.1. Let X be a Banach space, and let F be one of the two spaces defined above. Then the set of the well-posed problems in $\mathscr{F}$ has a $\sigma$-porous complement in $(\mathscr{F}, d)$.

Now we have a result concerning continuous/lower semicontinuous coercive functions. 


\section{Generic well-posedness in minimization problems}

Let $(X, \rho)$ be a complete metric space, $\psi$ a coercive function on $X$, and $\mathscr{F}$ one of the following two spaces of functions on $X$ :

(c) $\operatorname{LSC}(X, \psi)$, the collection of lower semicontinuous functions satisfying $f(x) \geq$ $\psi(x)$ for any $x \in X$

(d) $C(X, \psi)$, the collection of continuous functions on $X$ satisfying $f(x) \geq \psi(x)$ for any $x \in X$.

Observe that the two classes above consist of bounded from below functions $f$ for which the sets $f^{r}$ are bounded for every $r>0$. Both classes are complete metric spaces.

We then have the following theorem.

Theorem 4.2. Let F be one of the two spaces defined above. Then the set of the well-posed problems in $\mathscr{F}$ has a $\sigma$-porous complement in $(\mathscr{F}, d)$.

We remark that the uniform growth condition $f(x) \geq \psi(x)$ in the above result cannot be dropped. Consider, for example, the space of all continuous functions $f \geq 0$, on a normed space $X$, endowed with the above metric $d$ (obviously a complete metric space). Set

$$
U_{n}=\left\{f: \inf _{\|x\|>n} f<\inf _{\|x\| \leq n} f+\frac{1}{n}\right\}, \quad U=\bigcap_{n=1}^{\infty} U_{n} .
$$

Clearly, $f$ is ill-posed if $f \in U$. Moreover, it is easy to see that each $U_{n}$ is open and dense in the class; thus, no porosity (not even genericity) result holds in this case.

We now turn to constrained problems, and start with the usual convex programming problem:

$$
\text { minimize } f_{0}(x) \text { subject to } f_{1}(x) \leq 0, \ldots, f_{l}(x) \leq 0, x \in X \text {, }
$$

where $f_{i}, i=0, \ldots, l, l \geq 1$, are real-valued convex continuous functions defined on a Banach space $X$. (We have already provided a genericity result in this setting, see Theorem 3.2.)

The data space $\mathscr{A}$ will be a subspace of the Cartesian product of $(l+1)$ copies of $\operatorname{Conv}(X)$, endowed with the box metric:

$$
d\left[\left(f_{0}, \ldots, f_{l}\right),\left(g_{0}, \ldots, g_{l}\right)\right]=\max _{i=0, \ldots, l} d\left(f_{i}, g_{i}\right),
$$

where $d$ on the right-hand side stands for the same metric as earlier.

Let $a=\left(f_{0}, f_{1}, \ldots, f_{l}\right) \in[\operatorname{Conv}(X)]^{l+1}$. The feasible set of the problem determined by $a$ is, as usual, the set

$$
\mathscr{F}(a)=\left\{x \in X: f_{i}(x) \leq 0, \forall i=1, \ldots, l\right\}
$$

We define the data space as the collection of all $a \in[\operatorname{Conv}(X)]^{l+1}$ for which $\mathscr{F}(a) \neq \varnothing$. It is easy to see that the space $(\mathscr{A}, d)$ contains an open $\left(\right.$ in $\left.[\operatorname{Conv}(X)]^{l+1}\right)$ set which is dense in $\mathscr{A}$. Since $\left([\operatorname{Conv}(X)]^{l+1}, d\right)$ is a complete metric space, this implies that $(\mathscr{A}, d)$ is a Baire space. 
The function $f_{a}$ associated with $a \in \mathscr{A}$ is then defined in a standard way:

$$
f_{a}(x)=f_{0}(x) \quad \text { if } x \in F(a), \quad f_{a}(x)=\infty \quad \text { otherwise. }
$$

Theorem 4.3. Let $A$ be the space of constrained convex problems described above. Then the set of the well-posed problems in $\mathscr{A}$ has a $\sigma$-porous complement in $(\mathscr{A}, d)$.

The proof of these results (and of the following) relies on Corollary 2.9 to our variational principle. It is then necessary to single out at first what are the sets $\mathscr{A}_{m}, m \geq 0$.

The set $\mathscr{A}_{0}$, usually, is specific for the class under consideration and, inside it, we put "pathological" situations. For instance, for the convex/quasiconvex cases, we consider in $\mathscr{A}_{0}$ all the functions which are either lower unbounded or with unbounded level sets.

Instead, the sets $\mathscr{A}_{m}, m \geq 1$, are in general more or less the same in all cases, as well as the techniques to prove the condition of the principle relatively to $\mathscr{A}_{m}$. Just to give an example, we consider the convex case. Here the data space $A$ is identified with the family of convex continuous functions defined on a Banach space $X$. The set $\mathscr{A}_{m}$ is

$$
\mathscr{A}_{m}=\left\{f \in \mathscr{A} \backslash \mathscr{A}_{0}: f^{r} \bigcap B(0, m) \neq \varnothing, \forall r>0\right\} .
$$

Having an element $f \in \mathscr{A}_{m}$, we consider $\bar{x}$ such that

$$
\|\bar{x}\| \leq m, \quad f(\bar{x}) \leq \inf f+r,
$$

with suitable $r>0$. We next define a perturbation of the function $f$ :

$$
\bar{f}(x)=f(x)+\delta\|x-\bar{x}\|, \quad x \in X,
$$

with suitable $\delta$. Inasmuch as $\bar{x}$ almost minimizes $f$, the term $\delta\|x-\bar{x}\|$ creates a kind of well in such a way that the level sets (at small height) of the functions around $\bar{f}$ all remain in a small ball, and this is exactly what the principle requires. This happens as, roughly speaking, in one case, convexity/quasiconvexity, in the other cases, the coercivity assumption, guarantees that the level sets of close by functions all remain in a ball (a little larger than $B(0, m))$. Inside this ball the convergence is uniform, and this allows to control the behavior of close-by functions. Of course, in the constrained cases the situation is complicated by the fact that there are constraints to be fulfilled, but the basic idea remains the same. Thus, the ideas for the proof mimic those explained when commenting Theorem 3.2: the key point however is that porosity requires a form of "uniformity" in the estimations that makes necessary to cut the space $\mathscr{A}$ in the slices $\mathscr{A}_{m}$.

Our next result deals with a much more specific class of problems, namely quadratic programming problems in the $N$-dimensional Euclidean space $\mathbb{R}^{N}$, that is problems of the form

$$
\begin{aligned}
& \operatorname{minimize}\left\langle Q_{0} x, x\right\rangle+\left\langle c_{0}, x\right\rangle \\
& \quad \text { subject to }\left\langle Q_{1} x, x\right\rangle+\left\langle c_{1}, x\right\rangle \leq \alpha_{1}, \ldots,\left\langle Q_{l} x, x\right\rangle+\left\langle c_{l}, x\right\rangle \leq \alpha_{l}, x \in \mathbb{R}^{N},
\end{aligned}
$$

where $Q_{i}$ are $N \times N$ symmetric matrices, $c_{i} \in \mathbb{R}^{N},\langle\cdot, \cdot\rangle$ is the usual scalar product in $\mathbb{R}^{N}$, and $\alpha_{i} \in \mathbb{R}$. 
Every such problem is determined by the $3 l+2$-uple

$$
a=\left(Q_{0}, \ldots, Q_{l}, c_{0}, \ldots, c_{l}, \alpha_{1}, \ldots, \alpha_{l}\right) .
$$

The distance between two uples, $a=\left(Q_{0}, \ldots, Q_{l}, c_{0}, \ldots, c_{l}, \alpha_{1}, \ldots, \alpha_{l}\right)$ and $b=\left(R_{0}, \ldots, R_{l}\right.$, $\left.d_{0}, \ldots, d_{l}, \beta_{1}, \ldots, \beta_{l}\right)$, is defined by

$$
d(a, b)=\max _{0 \leq i \leq l}\left\{\left\|Q_{i}-R_{i}\right\|, \| c_{i}-d_{i}||,\left|\alpha_{i}-\beta_{i}\right|\right\}
$$

where $\alpha_{0}=\beta_{0}=0$. The metric $d$ is then compatible with the uniform convergence on bounded sets of the functions

$$
f_{i}(x)=\left\langle Q_{i} x, x\right\rangle+\left\langle c_{i}, x\right\rangle-\alpha_{i}
$$

As a data space, we take

$$
\begin{aligned}
\mathscr{A}=\left\{a=\left(Q_{0}, \ldots, Q_{l}, c_{0}, \ldots, c_{l}, \alpha_{1}, \ldots, \alpha_{l}\right):\right. \\
\left.\mathscr{F}(a) \neq \varnothing, \max _{i=0, \ldots, l}\left\langle Q_{i} x, x\right\rangle \geq 0 \forall x \in \mathbb{R}^{N}\right\} .
\end{aligned}
$$

The requirement that the maximum of the quadratic forms be nonnegative is mandatory: if for some data $a$, there exists $\tilde{x} \in \mathbb{R}^{N}$ such that $\max _{i=0, \ldots, l}\left\langle Q_{i} \tilde{x}, \tilde{x}\right\rangle<0$, then $t \tilde{x} \in$ $\mathscr{F}(a)$ for $t>0$ large enough and hence, for all problems nearly $a$, the corresponding objective function is unbounded below on the feasible set. Therefore, even generic wellposedness is not possible outside the above fixed class.

Theorem 4.4. Let $(\mathscr{A}, d)$ be the class of the quadratic mathematical programming problems described above. Then the set of well-posed problems in $\mathcal{A}$ has $\sigma$-porous complement in $(\mathscr{A}, d)$.

Corollary 4.5. Let $\mathscr{A}$ be the class of the quadratic mathematical programming problems introduced above. Then the set of ill-posed problems in $\mathscr{A}$ is a set of Lebesgue measure zero in $\mathbb{R}^{(l+1)\left(N^{2}+N\right)+l}$.

The proof of this result (in particular, the problem of singling out the set $\mathscr{A}_{0}$ of the "pathological problems") is technically much more complicated than the others. This depends not only on the fact that we are dealing with a special (finite-dimensional) family of problems, but also that we must take into account problems for which no convexity or coercivity property holds.

The final results we will mention here are our most recent ones, still unpublished. Carrying on our program to deal with specific families of problems, we consider now again the convex programming problems, but allowing this time very special perturbations. The setting is as follows.

We are given Banach spaces $X, Y, Z$ such that $X$ is separable and reflexive, $Y$ and $Z$ are Banach spaces with separable duals. A closed convex cone $C \subset Y$ with nonempty interior is also given. Moreover, $f: X \rightarrow \mathbb{R}$ is a continuous convex function, $g: X \rightarrow Y$ is continuous and $C$-convex, and $L$ is a linear continuous operator with $L(X)=Z$. Given 
$(p, a, b) \in X^{*} \times Y \times Z$, the problem $(\mathbf{P}(p, a, b))$ is the following:

$(\mathbf{P}(p, a, b))$

$$
\text { minimize } f(x)-\langle p, x\rangle \quad \text { subject to } g(x) \leq a, L x=b \text {. }
$$

The inequality constraint $g(x) \leq a$ here refers to the ordering induced by the cone $C$.

Set

$$
\begin{aligned}
V(p, a, b) & =\inf \{f(x)-\langle p, x\rangle: g(x) \leq a, L x=b\}, \\
F_{a, b}(x) & = \begin{cases}f(x), & \text { if } g(x) \leq a, L x=b, \\
\infty, & \text { otherwise. }\end{cases}
\end{aligned}
$$

Thus the initial (constrained) minimum problem $(\mathbf{P}(p, a, b))$ is equivalent to the (unconstrained) problem of minimizing $F_{a, b}(\cdot)-\langle p, \cdot\rangle$. We have that

$$
F_{a, b}^{*}(p)=\sup _{x}\left(\langle p, x\rangle-F_{a, b}(x)\right)=-V(p, a, b),
$$

so that the value function $V$ is concave in $p$, for every $(a, b) \in Y \times Z$, and convex in $(a, b)$, for every $p \in X^{*}$. Denoting by $S(p, a, b)$ the multifunction that to the given triple $(p, a, b)$ associates the solution set of $(\mathbf{P}(p, a, b))$, we then have that $S(p, a, b)=\partial F_{a, b}^{*}(p)$. Moreover, the Lagrange multiplier multifunction $\Lambda(p, a, b)$ is such that $\Lambda(p, a, b)=\partial F_{(\cdot, \cdot)}^{*}(p)(a, b)$. Thus we have the fundamental formula

$$
S(p, a, b) \times \Lambda(p, a, b)=\partial V(p, a, b) .
$$

(We remind that the subdifferential of a concave/convex function $h$ is defined as follows:

$$
\partial h(x, y)=\{(p, q): p \in \partial(-h)(\cdot, y)(x), q \in \partial h(x, \cdot)(y)\},
$$

and it is a maximal monotone operator.)

The set of the meaningful problems is defined by

$$
M=\left\{(p, a, b) \in X^{*} \times Y \times Z:|V(p, a, b)|<\infty\right\} .
$$

We need some assumption in order to assure that the set of the meaningful problems is big enough, that is, it contains an open set.

So, let $C^{+}=\left\{y^{*} \in Y^{*}:\left\langle y^{*}, y\right\rangle \geq 0\right.$, for all $\left.y \in C\right\}$ be the dual cone to $C$, and set

$$
\gamma(x)=\max \left\{f(x), \sup _{y^{*} \in C^{+} \cap B}\left\langle y^{*}, g(x)\right\rangle\right\}
$$

( $B$ denotes the unit ball in $Y^{*}$ ). Then we assume that

(G)

$$
\lim _{\|x\| \rightarrow \infty, x \in \operatorname{ker} L} \gamma(x)=\infty
$$


We now provide two definitions, the first one of $\sigma$-porosity, the other one of wellposedness, suited to this special setting. Observe that here we need to use a weaker concept of $\sigma$-porosity than that previously introduced, however, the nice properties of the $\sigma$-porous sets we described before are also enjoyed by the sets fulfilling this weaker form. On the other hand, we use here a very strong concept of well-posedness.

Definition 4.6. Let $M$ be a metric space, and let $A \subset M$. Let $x \in M, R>0$ and denote by $\sigma(x, A, R)$ the supremum of all $r>0$ such that there exists $z \in M$ such that $B(z, r) \subset$ $B(x, R) \backslash A$. The number $\lim \sup _{R \rightarrow 0}(\sigma(x, A, R) / R)$ is called the porosity of $A$ at $x$. A set $A$ is said to be porous if the porosity at $x$ is positive for every $x \in A$. A set is called $\sigma$-porous if it is a countable union of porous sets.

Finally, we introduce the new well-posedness idea, making sense in the context of mathematical programming, as it involves also the Lagrange multipliers, that carry useful information on the problem.

Definition 4.7. The problem $(\mathbf{P}(p, a, b))$ is said to be very well-posed if

(1) $(\mathbf{P}(p, a, b))$ is well-posed;

(2) there is a unique Lagrange multiplier for $(\mathbf{P}(p, a, b))$;

(3) if $\left(p_{n}, a_{n}, b_{n}\right) \rightarrow(p, a, b)$ if $\lambda_{n} \in \Lambda\left(p_{n}, a_{n}, b_{n}\right)$, then $\lambda_{n} \rightarrow \lambda$.

In the language of multifunctions, the last condition amounts to saying that the Lagrange multiplier multifunction is upper semicontinuous, as easily seen.

We now set our first result.

Theorem 4.8. Let $X$ be a reflexive, separable Banach space, let $Y, Z$ be Banach spaces with separable duals. Assume the coercivity condition $(\mathbf{G})$ and that $L$ is onto. Then the collection of $(p, a, b) \in \mathcal{M}$ such that $(\mathbf{P}(p, a, b))$ is not very well-posed is $\sigma$-porous in $M$.

In the finite-dimensional case, we can obtain another interesting result, that is, not only the majority of the problems are very well-posed, but also the (solution, Lagrange multiplier) multifunction enjoys, for most problems, a sort of Lipschitz stability property. To see this, we provide some further definition. Assuming $X$ and $Y$ are Banach spaces and $G: X \rightarrow Y$ is a multivalued function.

Definition 4.9. $G$ is said to be Lipschitz stable at $x \in X$ if $G(x)$ is a singleton and if there are a neighborhood $\mathcal{N}$ of $x$ and $k>0$ such that, for $u \in \mathcal{N}$ and $y \in G(u)$, the following holds:

$$
\|y-G(x)\| \leq k\|u-x\| .
$$

Theorem 4.10. Let $X, Y$ be Euclidean spaces and let $(\mathbf{G})$ hold. Then the set of the parameters $(p, a, b) \in X \times Y \times \operatorname{Im} L$ such that the problem $(\mathbf{P}(p, a, b))$ is meaningful but either is not very well-posed, or the (solution, Lagrange multiplier) multifunction $S(\cdot, \cdot, \cdot) \times \Lambda(\cdot, \cdot, \cdot)$ is not Lipschitz stable at $(p, a, b)$ is a set of Lebesgue measure zero in $X \times Y \times \operatorname{Im} L$.

These results are very nontrivial even in the finite-dimensional case.

Proofs of these results are based on three fundamental theorems of convex analysis: the Asplund-Rockafellar theorem [1] about duality between rotundity and Fréchet 
differentiability, the Preiss-Zajíček [16] theorem on Fréchet differentiability, up to a $\sigma$ porous set, of a convex continuous function on a space with separable dual (which we extend to concave/convex functions), and the theorem of Mignot [15] about almost everywhere differentiability of a finite-dimensional maximal monotone operator.

\section{Acknowledgments}

This paper has its roots in a visit of the second author to the Department of Mathematics, Technion, Haifa, during June 2003. The nice hospitality provided by the Department is gratefully acknowledged. The research was supported in part by the US-Israel Binational Fund under the Grant 2000157. The research of the second author was partially supported by Ministero dell'Istruzione, dell'Università e della Ricerca (COFIN 2001).

\section{References}

[1] E. Asplund and R. T. Rockafellar, Gradients of convex functions, Trans. Amer. Math. Soc. 139 (1969), 443-467.

[2] H. Attouch, R. E. Lucchetti, and R. J.-B. Wets, The topology of the $\rho$-Hausdorff distance, Ann. Mat. Pura Appl. (4) 160 (1992), 303-320.

[3] G. Beer and R. E. Lucchetti, Minima of quasi-convex functions, Optimization 20 (1989), no. 5, 581-596.

[4] Convex optimization and the epi-distance topology, Trans. Amer. Math. Soc. 327 (1991), no. $2,795-813$.

[5] - The epi-distance topology: continuity and stability results with applications to convex optimization problems, Math. Oper. Res. 17 (1992), no. 3, 715-726.

[6] F. S. de Blasi, J. Myjak, and P. L. Papini, Porous sets in best approximation theory, J. London Math. Soc. (2) 44 (1991), no. 1, 135-142.

[7] R. Deville and J. P. Revalski, Porosity of ill-posed problems, Proc. Amer. Math. Soc. 128 (2000), no. 4, 1117-1124.

[8] A. L. Dontchev and T. Zolezzi, Well-Posed Optimization Problems, Lecture Notes in Mathematics, vol. 1543, Springer, Berlin, 1993.

[9] A. D. Ioffe and R. E. Lucchetti, Generic existence, uniqueness and stability in optimization problems, Nonlinear Optimization and Related Topics (Erice, 1998) (G. Di Pillo and F. Giannessi, eds.), Appl. Optim., vol. 36, Kluwer Academic, Dordrecht, 2000, pp. 169-182.

[10] A. D. Ioffe, R. E. Lucchetti, and J. P. Revalski, A variational principle for problems with functional constraints, SIAM J. Optim. 12 (2001/2002), no. 2, 461-478.

[11] A. D. Ioffe, R. E. Lucchetti, and J. P. Revalski, Almost every convex or quadratic programming problem is well posed, Math. Oper. Res. 29 (2004), no. 2, 369-382.

[12] A. D. Ioffe and V. M. Tikhomirov, Some remarks on variational principles, Math. Notes 61 (1997), no. 1-2, 248-253.

[13] A. D. Ioffe and A. J. Zaslavski, Variational principles and well-posedness in optimization and calculus of variations, SIAM J. Control Optim. 38 (2000), no. 2, 566-581.

[14] R. E. Lucchetti and F. Patrone, Sulla densità e genericità di alcuni problemi di minimo ben posti, Boll. Un. Mat. Ital. B (5) 15 (1978), no. 1, 225-240 (Italian).

[15] F. Mignot, Contrôle dans les inéquations variationelles elliptiques, J. Funct. Anal. 22 (1976), no. 2, 130-185 (French).

[16] D. Preiss and L. Zajíček, Fréchet differentiation of convex functions in a Banach space with a separable dual, Proc. Amer. Math. Soc. 91 (1984), no. 2, 202-204.

[17] S. Reich and A. J. Zaslavski, Generic convergence of descent methods in Banach spaces, Math. Oper. Res. 25 (2000), no. 2, 231-242. 
[18] The set of divergent descent methods in a Banach space is $\sigma$-porous, SIAM J. Optim. 11 (2001), no. 4, 1003-1018.

[19] Well-posedness and porosity in best approximation problems, Topol. Methods Nonlinear Anal. 18 (2001), no. 2, 395-408.

[20] J. E. Spingarn and R. T. Rockafellar, The generic nature of optimality conditions in nonlinear programming, Math. Oper. Res. 4 (1979), no. 4, 425-430.

[21] L. Zajíček, Porosity and $\sigma$-porosity, Real Anal. Exchange 13 (1987/1988), no. 2, 314-350.

[22] A. J. Zaslavski, Generic well-posedness of optimal control problems without convexity assumptions, SIAM J. Control Optim. 39 (2000), no. 1, 250-280.

[23] T. Zolezzi, Well-posedness criteria in optimization with application to the calculus of variations, Nonlinear Anal. 25 (1995), no. 5, 437-453.

[24] _ Extended well-posedness of optimization problems, J. Optim. Theory Appl. 91 (1996), no. 1, 257-266.

A. Ioffe: Department of Mathematics, Technion - Israel Institute of Technology, 32000 Haifa, Israel E-mail address: ioffe@math.technion.ac.il

R. E. Lucchetti: Dipartimento di Matematica, Politecnico di Milano, Via Bonardi 7, 20133 Milano, Italy

E-mail address: robluc@mate.polimi.it 


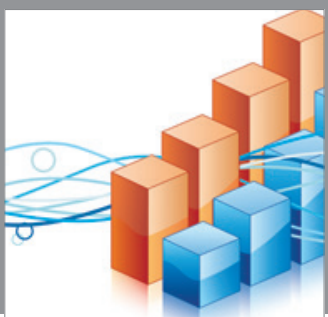

Advances in

Operations Research

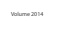

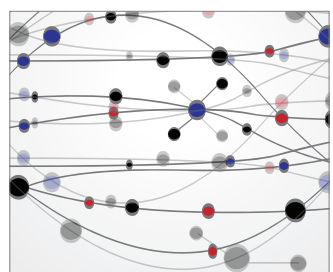

\section{The Scientific} World Journal
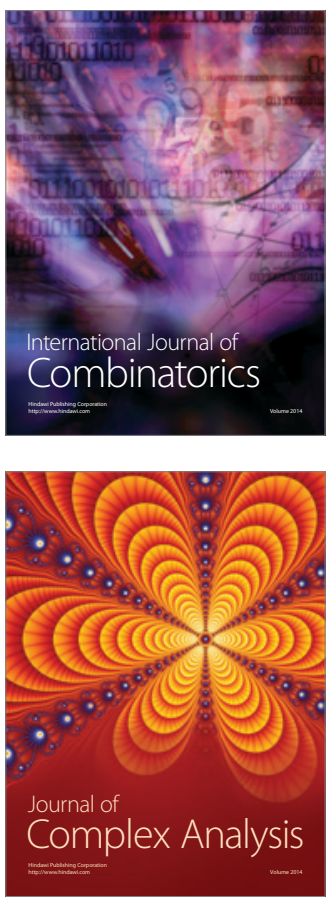

International Journal of

Mathematics and

Mathematical

Sciences
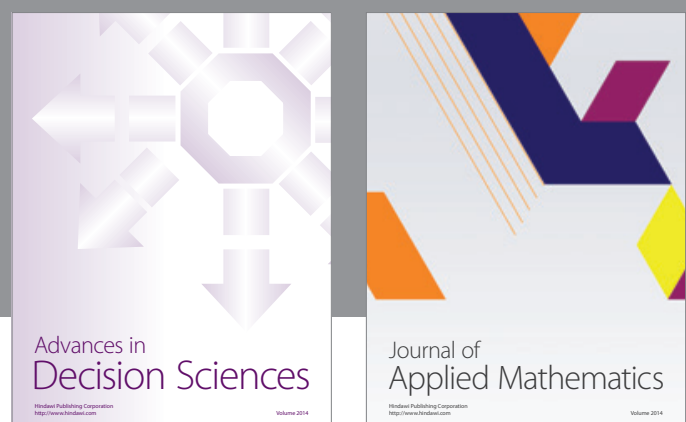

Journal of

Applied Mathematics
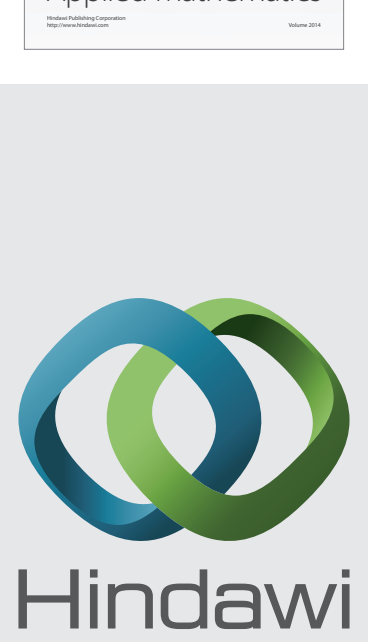

Submit your manuscripts at http://www.hindawi.com
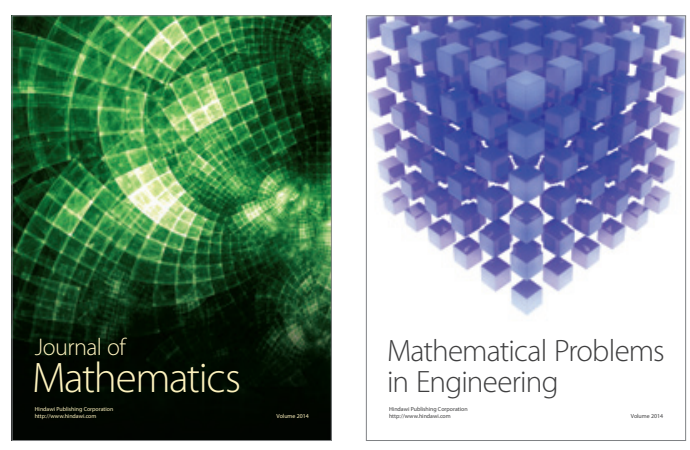

Mathematical Problems in Engineering
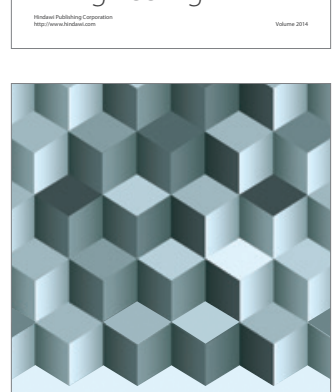

Journal of

Function Spaces
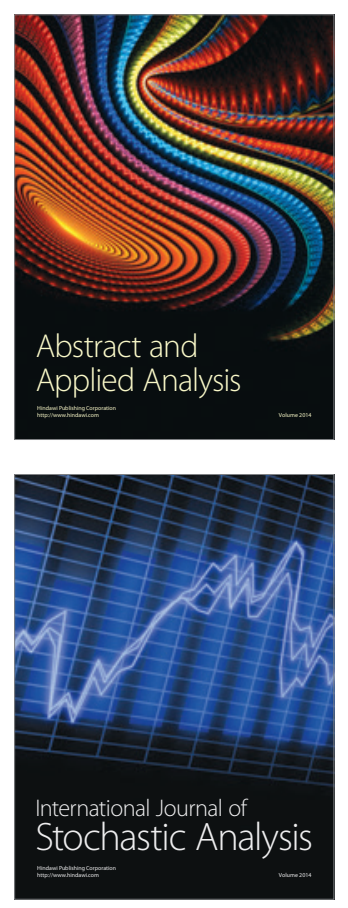

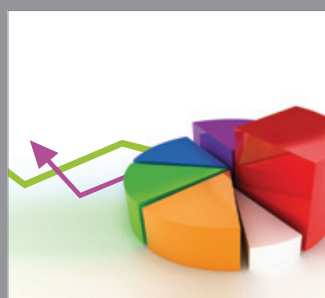

ournal of

Probability and Statistics

Promensencen
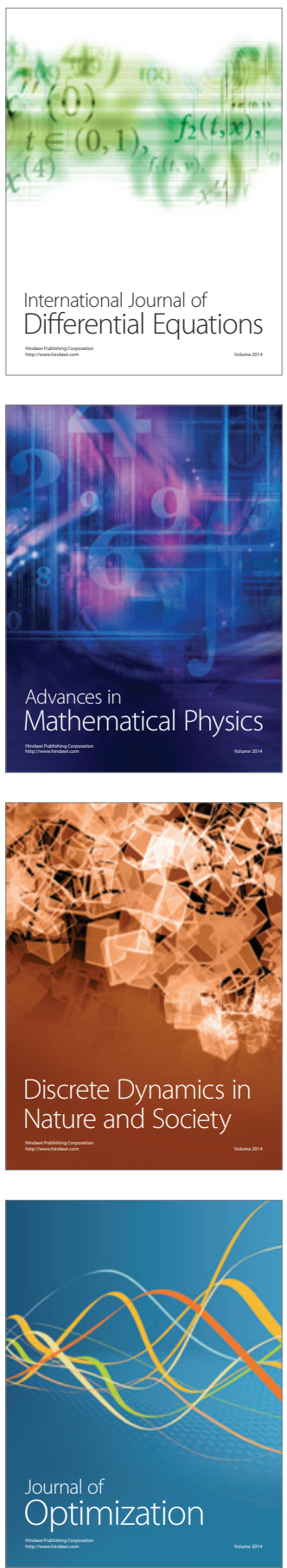